\title{
Protocol
}

\section{Dissection, Plating, and Maintenance of Cortical Astrocyte Cultures}

\author{
Cristóvão Albuquerque, Donald J. Joseph, Papiya Choudhury, and Amy B. MacDermott ${ }^{1}$ \\ Department of Physiology and Cellular Biophysics and Department of Neuroscience, Columbia University, New York, \\ NY 10032, USA
}

\section{INTRODUCTION}

Survival of central nervous system neurons in culture is usually greatly improved if the neurons are plated on top of a layer of confluent astrocytes. Under these conditions, neurons attach more firmly and develop a more readily identifiable bidimensional neuritic tree than in culture conditions where primary cells are plated on top of collagen, laminin, or other cell-free substrates. Although dissociated cells from any central nervous system region will include astrocytes and other glia, these generally do not support the survival of the primary neurons as well as preplated astrocyte feeder layers. For example, plating dorsal root ganglion (DRG)/dorsal horn (DH) cocultures directly onto collagen or laminin results in a very low number of attached $\mathrm{DH}$ neurons, and those have stunted neurites. Furthermore, although a higher number of DRG neurons do attach without astrocytes, this attachment is weaker, as demonstrated by the tendency of those neurons (or at least their neurites) to blow off the coverslip when a local superfusion system is directed at them. Neonatal rat cortex provides abundant astrocytes that can be cultured readily. The protocol presented here selects for type I astrocytes that grow in a monolayer with contact inhibition.

\section{RELATED INFORMATION}

For specific protocols describing other techniques used in the preparation of neuronal cultures, see Preparation of Coverslips for Neuronal Cultures (Albuquerque et al. 2009a), Dissection, Plating, and Maintenance of Dorsal Horn Neuron Cultures (Albuquerque et al. 2009b), and Dissection, Plating, and Maintenance of Dorsal Root Ganglion Neurons for Monoculture and for Coculture with Dorsal Horn Neurons (Albuquerque et al. 2009c).

\section{MATERIALS}

CAUTIONS AND RECIPES: Please see Appendices for appropriate handling of materials marked with <!>, and recipes for reagents marked with $<R>$.

\section{Reagents}

All solutions must be sterile.

$<$ !>Cytosine $\beta$-D-arabinofuranoside (Ara-C; $1 \mathrm{mM}$, prepared in MEM; Sigma C1768)

Dulbecco's phosphate-buffered saline (D-PBS; Invitrogen 14190144)

Ethanol (70\% [in a squirt bottle] and 95\%)

$<$ R $>$ IMDM for astrocytes

${ }^{1}$ Corresponding author (abm1@columbia.edu) 


\section{$<$ ! Isofluorane \\ Leibovitz's L-15 medium (ice-cold) (Invitrogen 11415064) \\ Minimum essential medium (MEM; Invitrogen 11090081) \\ Modified Eagle medium (S-MEM; Invitrogen 11380037) \\ Rat pups, 0- to 1-d old (i.e., 21-22 d from the plug date) \\ $<$ ! Trypsin (2.5\%; Invitrogen 15090046) \\ $<$ !>Trypsin-EDTA (0.05\%; Invitrogen 25300054)}

\section{Equipment}

Beaker $(100 \mathrm{~mL})$

Centrifuge (culture)

Container (airtight)

Cotton balls

Coverslips (poly-D-lysine [PDL]- and laminin-coated, in 35-mm dishes) (for mass culture)

Coverslips (with agarose-collagen microislands, in 35-mm dishes) (for microisland culture) Prepare as described in Preparation of Coverslips for Neuronal Cultures (Albuquerque et al. 2009a).

Dishes (culture, plastic, 35- and 60-mm) (BD Falcon 351008 and 351007, respectively)

Flasks (culture, rectangular, $75-\mathrm{cm}^{2}$, canted-neck, equipped with vent cap) (Corning Life Sciences 430641)

Forceps (fine)

Fume hood

Hemacytometer

Ice

Laboratory tissue

Mammalian cell culture incubator preset to $37^{\circ} \mathrm{C}$ (water-jacketed, equilibrated with $5 \% \mathrm{CO}_{2}$ )

Micropipettor with tips

Microscope (phase-contrast)

Paper towels

Pasteur pipettes (sterile)

Flame-polish the tips of the pipettes with a Bunsen burner before use.

Scissors (large and small iridectomy)

Shaker preset to $37^{\circ} \mathrm{C}$

Spatula (small)

Tubes (capped conical centrifuge, sterile, $15 \mathrm{~mL}$ ) (BD Falcon 352096)

Tubes (capped conical centrifuge, sterile, $50 \mathrm{~mL}$ ) (Corning 430828)

Tweezers

\section{METHOD}

\section{Brain Dissection}

1. Before beginning dissection, place three to five $60-\mathrm{mm}$ culture dishes containing cold L-15 medium on ice.

2. For each animal to be dissected, add $2 \mathrm{~mL}$ S-MEM to a 35- $\mathrm{mm}$ dish. Place the dishes in the culture incubator.

3. Cover the bottom of a $100-\mathrm{mL}$ beaker with paper towels. Fill the beaker with $95 \%$ ethanol. Place the dissection instruments in the beaker.

Lining the beaker with paper towels protects the tips of the fine forceps.

4. Anesthetize a rat pup by placing it in a small closed container with an isofluorane-soaked cotton ball until loss of righting reflex and pinch response occurs.

Perform the anesthesia under a fume hood. 
5. Remove the pup from the container. Wipe the pup's neck with $70 \%$ ethanol. Cut off the head.

6. Holding the head with a laboratory tissue, wash the head with $70 \%$ ethanol from a squirt bottle.

7. Cut open the skin longitudinally with the large iridectomy scissors, exposing the whole superior surface of the skull.

8. Carefully cut open the skull longitudinally with the scissors. Use tweezers to pull open the two halves to expose the brain (Fig. 1A).

9. Carefully scoop the brain out of the skull with a spatula.

10. Place the brain into a $60-\mathrm{mm}$ dish containing ice-cold L-15 medium (see Fig. 1C).

11. Cut off the brainstem.

12. Separate the two hemispheres with scissors.

13. Scoop the inside of the hemispheres with the spatula, leaving only the neocortical halves. Only the convexity of the hemispheres should remain.

14. Remove the meninges from the surface of the cortical halves using fine forceps and small scissors (Fig. 2).

15. Place the two hemispheres into the $35-\mathrm{mm}$ dish containing $2 \mathrm{~mL}$ of prewarmed S-MEM (from Step 2). Mince the tissue with the small iridectomy scissors.

16. Add $200 \mu \mathrm{L}$ of trypsin. Return the dish to the incubator. Incubate for 20-25 min.

17. Add $2 \mathrm{~mL}$ of IMDM for astrocytes to each dish.

18. Transfer the whole sample $(\sim 4 \mathrm{~mL})$ to a $15-\mathrm{mL}$ centrifuge tube. Dissociate the cells by gently aspirating and expelling the minced tissue two to four times with a sterile, flame-polished Pasteur pipette.

19. Pellet the cells by centrifugation at $1300 \mathrm{rpm}$ for $8 \mathrm{~min}$. Discard the supernatant.

20. Add $2 \mathrm{~mL}$ of IMDM for astrocytes to the pellet. Disperse the pellet by gently aspirating and expelling it through a sterile, flame-polished Pasteur pipette.

21. Transfer the resuspended cells to a culture flask. Add $11 \mathrm{~mL}$ of IMDM for astrocytes to the cells. Return the flask to the incubator.

22. Monitor the growth of the astrocytes by phase-contrast microscopy. Astrocytes grown to confluency are identifiable as a monolayer of large, flat cells in a "brick wall" arrangement.

\section{Astrocyte Splitting}

Shaking the culture removes undesirable cell populations such as cortical neurons and other glial subtypes. Adding the mitotic inhibitor Ara-C kills rapidly dividing, unwanted cell populations such as fibroblasts. Because Ara-C is toxic to neurons, it also assists in eliminating cortical neurons. After shaking in Ara-C, the remaining attached cells are lifted from the bottom of the flask and either plated directly onto coverslips or into a fresh flask.

23. Add $130 \mu \mathrm{L}$ of Ara-C to each flask. Close the cap tightly.

24. Place the flask in a heated shaker (or in a shaker in an incubator or hot room at $37^{\circ} \mathrm{C}$ ). Shake for 6-7 h.

The speed of rotation should be high, but not so much that the medium will splash.

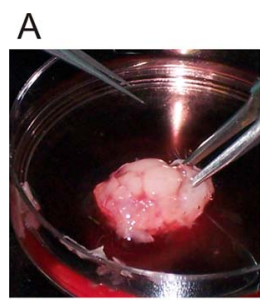

$\mathrm{B}$

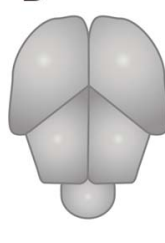

C

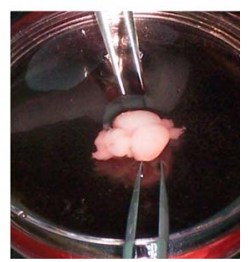

FIGURE 1. Removing the brain. (A) The rat pup brain is still in the skull, but the top flaps have been removed. (B) Diagram of rat brain. The cortical hemispheres are at the top. (C) The brain has been removed from the skull. The severed optic tracts (right) and the brainstem (left) are visible. 
A

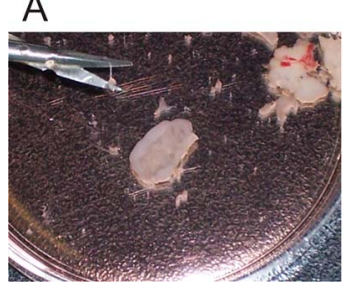

B

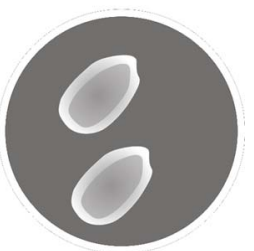

FIGURE 2. (A) Rat cortex isolated and cleaned of meninges. (B) Diagram of two cortices.

25. Remove the medium from the flask. Wash the cells once with D-PBS.

26. Add $5 \mathrm{~mL}$ of trypsin-EDTA to the flask. Incubate for $5-10 \mathrm{~min}$ at $37^{\circ} \mathrm{C}$.

27. Monitor the flask by holding it up to the light to see whether the astrocytes have lifted off the bottom.

The solution should be cloudy with cells in suspension. If cells remain stuck to the bottom, incubate longer and/or gently tap the sides of the flask.

28. Add $13 \mathrm{~mL}$ of fresh IMDM for astrocytes to the flask. Transfer the whole suspension $(\sim 18 \mathrm{~mL})$ to a 50-mL centrifuge tube.

29. Centrifuge at $1300 \mathrm{rpm}$ for $8 \mathrm{~min}$. Remove the supernatant.

30. Add $5 \mathrm{~mL}$ of fresh IMDM for astrocytes to the pellet. Disperse the cells gently by repeatedly pipetting the medium.

31. Add $8 \mathrm{~mL}$ of IMDM for astrocytes to the tube. Gently mix the cells by repeated pipetting. Transfer to a fresh flask.

32. Maintain the astrocytes in culture by feeding every 1-2 wk with $13 \mathrm{~mL}$ of IMDM for astrocytes. Once the astrocytes have grown to confluency again, they are ready to be plated onto coverslips.

\section{Astrocyte Plating}

33. Lift cells as described in Steps 25-30.

34. Count the cells with a hemacytometer:

i. For mass culture, plate $3.6 \times 10^{4}$ cells per PDL- and laminin-coated coverslip in each 35-mm dish.

ii. For microisland cultures, plate $1.2 \times 10^{4}$ cells per microisland-containing coverslip in each 35-mm dish.

35. Bring the final volume in each dish to $2 \mathrm{~mL}$ with IMDM for astrocytes. Place the dishes in the incubator until use.

Figure 3 shows confluent astrocytes on a single microisland. Astrocyte-plated coverslips should be used within 15-20d.

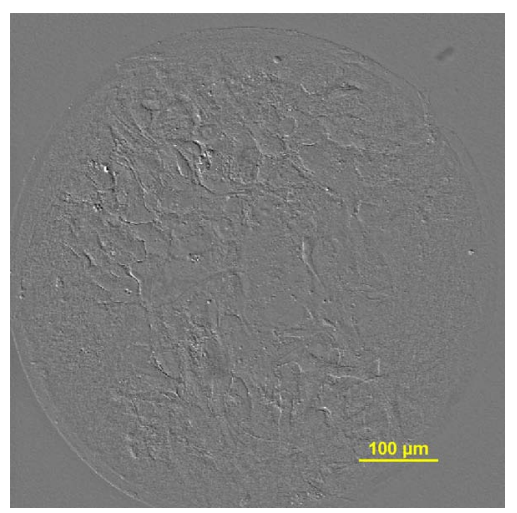

FIGURE 3. Photomicrograph of a microisland with attached, confluent astrocytes viewed under differential interference contrast optics. 


\section{DISCUSSION}

The quality of the astrocyte layer is the main determinant of the success of the neuronal cultures. Healthy astrocytes on the coverslip are a good predictor of successful neuronal attachment, robust neuritic outgrowth, and cultures that will last up to $4-5 \mathrm{wk}$ in the incubator. Young astrocytes (i.e., one passage) seem to give better results.

\section{REFERENCES}

Albuquerque C, Joseph D], Choudhury P, MacDermott AB. 2009a. Preparation of coverslips for neuronal cultures. Cold Spring Harb Protoc (this issue). doi: $10.1101 /$ pdb.prot5272.

Albuquerque C, Joseph DJ, Choudhury P, MacDermott AB. 2009b. Dissection, plating, and maintenance of dorsal horn neuron cultures.

Cold Spring Harb Protoc (this issue). doi: 10.1101/pdb.prot5274. Albuquerque C, Joseph D], Choudhury P, MacDermott AB. 2009c. Dissection, plating, and maintenance of dorsal root ganglion neurons for monoculture and for coculture with dorsal horn neurons. Cold Spring Harb Protoc (this issue). doi: 10.1101/pdb.prot5275. 


\section{Dissection, Plating, and Maintenance of Cortical Astrocyte Cultures}

Cristóvão Albuquerque, Donald J. Joseph, Papiya Choudhury and Amy B. MacDermott

Cold Spring Harb Protoc; doi: 10.1101/pdb.prot5273

\begin{tabular}{|c|c|}
\hline $\begin{array}{l}\text { Email Alerting } \\
\text { Service }\end{array}$ & Receive free email alerts when new articles cite this article - click here. \\
\hline $\begin{array}{l}\text { Subject } \\
\text { Categories }\end{array}$ & $\begin{array}{l}\text { Browse articles on similar topics from Cold Spring Harbor Protocols. } \\
\text { Cell Biology, general (1382 articles) } \\
\text { Cell Culture (301 articles) } \\
\text { Cell Imaging (525 articles) } \\
\text { Imaging for Neuroscience (342 articles) } \\
\text { Neural Cell Culture (57 articles) } \\
\text { Neuroscience, general (357 articles) }\end{array}$ \\
\hline
\end{tabular}

